\title{
The Fundamental Group of Balanced Simplicial Complexes and Posets
}

\author{
Steven Klee \\ Department of Mathematics, Box 354350 \\ University of Washington, Seattle, WA 98195-4350, USA, \\ klees@math . washington. edu \\ Submitted: Sep 29, 2008; Accepted: Apr 18, 2009; Published: Apr 27, 2009 \\ Mathematics Subject Classifications: 05E25, 06A07, 55U10 \\ Dedicated to Anders Björner on the occasion of his 60th birthday
}

\begin{abstract}
We establish an upper bound on the cardinality of a minimal generating set for the fundamental group of a large family of connected, balanced simplicial complexes and, more generally, simplicial posets.
\end{abstract}

\section{Introduction}

One commonly studied combinatorial invariant of a finite $(d-1)$-dimensional simplicial complex $\Delta$ is its $f$-vector $f=\left(f_{0}, \ldots, f_{d-1}\right)$ where $f_{i}$ denotes the number of $i$-dimensional faces of $\Delta$. This leads to the study of the $h$-numbers of $\Delta$ defined by the relation $\sum_{i=0}^{d} h_{i} \lambda^{d-i}=\sum_{i=0}^{d} f_{i-1}(\lambda-1)^{d-i}$. A great deal of work has been done to relate the $f$-numbers and $h$-numbers of $\Delta$ to the dimensions of the singular homology groups of $\Delta$ with coefficients in a certain field; see, for example, the work of Björner and Kalai in [2] and [3], and Chapters 2 and 3 of Stanley [13]. In comparison, very little seems to be known about the relationship between the $f$-numbers of a simplicial complex and various invariants of its homotopy groups. In this paper, we bound the minimal number of generators of the fundamental group of a balanced simplicial complex in terms of $h_{2}$. More generally, we bound the minimal number of generators of the fundamental group of a balanced simplicial poset in terms of $h_{2}$.

It was conjectured by Kalai [7] and proved by Novik and Swartz in [8] that if $\Delta$ is a $(d-1)$-dimensional manifold that is orientable over the field $\mathbf{k}$, then

$$
h_{2}-h_{1} \geq\left(\begin{array}{c}
d+1 \\
2
\end{array}\right) \beta_{1}
$$

where $\beta_{1}$ is the dimension of the singular homology group $H_{1}(\Delta ; \mathbf{k})$. The Hurewicz Theorem (see Spanier [10]) says that $H_{1}(X ; \mathbb{Z})$ is isomorphic to the abelianization of $\pi_{1}(X, *)$ 
for a connected space $X$. We will see below that $\pi_{1}(\Delta, *)$ is finitely generated. Thus the Hurewicz Theorem says that the minimal number of generators of the fundamental group of a simplicial complex $\Delta$ is greater than or equal to the number of generators of $H_{1}(\Delta ; \mathbb{Z})$. By the universal coefficient theorem, $H_{1}(\Delta ; \mathbf{k}) \approx H_{1}(\Delta ; \mathbb{Z}) \otimes \mathbf{k}$ for any field $\mathbf{k} ;$ and, consequently, the minimal number of generators of $\pi_{1}(\Delta, *)$ is greater than or equal to $\beta_{1}(\Delta)$ for any field $\mathbf{k}$.

In this paper, we study simplicial complexes and simplicial posets $\Delta$ that are pure and balanced with the property that every face $F \in \Delta$ of codimension at least 2 (including the empty face) has connected link. This includes the class of balanced triangulations of compact manifolds and, using the language of Goresky and MacPherson in [5], the more general class of balanced normal pseudomanifolds. Under these weaker assumptions, we show that

$$
h_{2} \geq\left(\begin{array}{l}
d \\
2
\end{array}\right) m(\Delta)
$$

where $m(\Delta)$ denotes the minimal number of generators of $\pi_{1}(\Delta, *)$.

The paper is structured as follows. Section 2 contains all necessary definitions and background material. In Section 3, we outline a sequence of theorems in algebraic topology that are used to give a description of the fundamental group in terms of a finite set of generators and relations. In Section 4, we use the theorems in Section 3 to prove Theorem 4.5. This theorem gives the desired bound on $m(\Delta)$. In Section 5, after giving some definitions related to simplicial posets, we extend the topological results in Section 3 and the result of Theorem 4.5 to the class of simplicial posets.

\section{Notation and Conventions}

Throughout this paper, we assume that $\Delta$ is a $(d-1)$-dimensional simplicial complex on vertex set $V=\left\{v_{1}, \ldots, v_{n}\right\}$. We recall that the dimension of a face $F \in \Delta$ is $\operatorname{dim} F=$ $|F|-1$, and the dimension of $\Delta$ is $\operatorname{dim} \Delta=\max \{\operatorname{dim} F: F \in \Delta\}$. A simplicial complex is pure if all of its facets (maximal faces) have the same dimension. The link of a face $F \in \Delta$ is the subcomplex

$$
\mathrm{lk}_{\Delta} F=\{G \in \Delta: F \cap G=\emptyset, F \cup G \in \Delta\} .
$$

Similarly, the closed star of a face $F \in \Delta$ is the subcomplex

$$
\overline{\mathrm{st}}_{\Delta} F=\{G \in \Delta: F \cup G \in \Delta\} \text {. }
$$

The geometric realization of $\Delta$, denoted by $|\Delta|$, is the union over all faces $F \in \Delta$ of the convex hull in $\mathbb{R}^{n}$ of $\left\{e_{i}: v_{i} \in F\right\}$ where $\left\{e_{1}, \ldots, e_{n}\right\}$ denotes the standard basis in $\mathbb{R}^{n}$. Given this geometric realization, we will make little distinction between the combinatorial object $\Delta$ and the topological space $|\Delta|$. For example, we will often abuse notation and write $H_{i}(\Delta ; \mathbf{k})$ instead of the more cluttered $H_{i}(|\Delta| ; \mathbf{k})$.

The $f$-vector of $\Delta$ is the vector $f=\left(f_{-1}, f_{0}, f_{1}, \ldots, f_{d-1}\right)$ where $f_{i}$ denotes the number of $i$-dimensional faces of $\Delta$. By convention, we set $f_{-1}=1$, corresponding to the empty 
face. If it is important to distinguish the simplicial complex $\Delta$, we write $f(\Delta)$ for the $f$-vector of $\Delta$, and $f_{i}(\Delta)$ for its $f$-numbers (i.e. the entries of its $f$-vector). Another important combinatorial invariant of $\Delta$ is the $h$-vector $h=\left(h_{0}, \ldots, h_{d}\right)$ where

$$
h_{i}=\sum_{j=0}^{i}(-1)^{i-j}\left(\begin{array}{l}
d-j \\
d-i
\end{array}\right) f_{j-1} .
$$

For us, it will be particularly important to study a certain class of complexes known as balanced simplicial complexes, which were introduced by Stanley in [11].

Definition 2.1 $A(d-1)$-dimensional simplicial complex $\Delta$ is balanced if its 1-skeleton, considered as a graph, is d-colorable. That is to say there is a coloring $\kappa: V \rightarrow[d]$ such that for all $F \in \Delta$ and distinct $v, w \in F$, we have $\kappa(v) \neq \kappa(w)$. We assume that a balanced complex $\Delta$ comes equipped with such a coloring $\kappa$.

The order complex of a rank- $d$ graded poset is one example of a balanced simplicial complex. If $\Delta$ is a balanced complex and $S \subseteq[d]$, it is often important to study the $S$-rank selected subcomplex of $\Delta$, which is defined as

$$
\Delta_{S}=\{F \in \Delta: \kappa(F) \subseteq S\}
$$

that is, for a fixed coloring $\kappa$, we define $\Delta_{S}$ to be the subcomplex of faces whose vertices are colored with colors from $S$. In [11] Stanley showed that

$$
h_{i}(\Delta)=\sum_{|S|=i} h_{i}\left(\Delta_{S}\right)
$$

\section{$3 \quad$ The Edge-Path Group}

In order to obtain a concrete description of $\pi_{1}(\Delta, *)$ that relies only on the structure of $\Delta$ as a simplicial complex, we introduce the edge-path group of $\Delta$ (see, for example, Seifert and Threlfall [9] or Spanier [10]). This will ultimately allow us to relate the combinatorial data of $f(\Delta)$ to the fundamental group of $\Delta$.

An edge in $\Delta$ is an ordered pair of vertices $\left(v, v^{\prime}\right)$ with $\left\{v, v^{\prime}\right\} \in \Delta$. An edge path $\gamma$ in $\Delta$ is a finite nonempty sequence $\left(v_{0}, v_{1}\right)\left(v_{1}, v_{2}\right) \cdots\left(v_{r-1}, v_{r}\right)$ of edges in $\Delta$. We say that $\gamma$ is an edge path from $v_{0}$ to $v_{r}$, or that $\gamma$ starts at $v_{0}$ and ends at $v_{r}$. A closed edge path at $v$ is an edge path $\gamma$ such that $v_{0}=v=v_{r}$.

We say that two edge paths $\gamma$ and $\gamma^{\prime}$ are simply equivalent if there exist vertices $v, v^{\prime}, v^{\prime \prime}$ in $\Delta$ with $\left\{v, v^{\prime}, v^{\prime \prime}\right\} \in \Delta$ such that the unordered pair $\left\{\gamma, \gamma^{\prime}\right\}$ is equal to one of the following unordered pairs:

- $\left\{\left(v, v^{\prime \prime}\right),\left(v, v^{\prime}\right)\left(v^{\prime}, v^{\prime \prime}\right)\right\}$,

- $\left\{\gamma_{1}\left(v, v^{\prime \prime}\right), \gamma_{1}\left(v, v^{\prime}\right)\left(v^{\prime}, v^{\prime \prime}\right)\right\}$ for some edge path $\gamma_{1}$ ending at $v$, 
- $\left\{\left(v, v^{\prime \prime}\right) \gamma_{2},\left(v, v^{\prime}\right)\left(v^{\prime}, v^{\prime \prime}\right) \gamma_{2}\right\}$ for some edge path $\gamma_{2}$ starting at $v^{\prime \prime}$,

- $\left\{\gamma_{1}\left(v, v^{\prime \prime}\right) \gamma_{2}, \gamma_{1}\left(v, v^{\prime}\right)\left(v^{\prime}, v^{\prime \prime}\right) \gamma_{2}\right\}$ for edge paths $\gamma_{1}, \gamma_{2}$ as above.

We note that the given vertices $v, v^{\prime}, v^{\prime \prime} \in \Delta$ need not be distinct. For example, $(v, v)$ is a valid edge (the edge that does not leave the vertex $v$ ), and we have the simple equivalence $\left(v, v^{\prime}\right)\left(v^{\prime}, v\right) \sim(v, v)$. We say that two edge paths $\gamma$ and $\gamma^{\prime}$ are equivalent, and write $\gamma \sim \gamma^{\prime}$, if there is a finite sequence of edge paths $\gamma_{0}, \gamma_{1}, \ldots, \gamma_{s}$ such that $\gamma=\gamma_{0}$, $\gamma^{\prime}=\gamma_{s}$ and $\gamma_{i}$ is simply equivalent to $\gamma_{i+1}$ for $0 \leq i \leq s-1$. It is easy to check that this defines an equivalence relation on the collection of edge paths $\gamma$ in $\Delta$ starting at $v$ and ending at $v^{\prime}$. Moreover, for two edge paths $\gamma$ and $\gamma^{\prime}$ with the terminal vertex of $\gamma$ equal to the initial vertex of $\gamma^{\prime}$, we can form their product edge path $\gamma \gamma^{\prime}$ by concatenation.

Now we pick a base vertex $v_{0} \in \Delta$. Let $E\left(\Delta, v_{0}\right)$ denote the set of equivalence classes of closed edge paths in $\Delta$ based at $v_{0}$. We multiply equivalence classes by $[\gamma] *\left[\gamma^{\prime}\right]=\left[\gamma \gamma^{\prime}\right]$ to give $E\left(\Delta, v_{0}\right)$ a group structure called the edge path group of $\Delta$ based at $v_{0}$.

The Cellular Approximation Theorem ([10] VII.6.17) tells us that any path in $\Delta$ is homotopic to a path in the 1-skeleton of $\Delta$. We use this fact to motivate the proof of the following theorem from Spanier.

Theorem 3.1 ([10] III.6.17) If $\Delta$ is a simplicial complex and $v_{0} \in \Delta$, then there is a natural isomorphism

$$
E\left(\Delta, v_{0}\right) \approx \pi_{1}\left(\Delta, v_{0}\right) .
$$

For a connected simplicial complex $\Delta$ we will also consider the group $G$, defined as follows. Let $T$ be a spanning tree in the 1-skeleton of $\Delta$. Since $\Delta$ is connected, such a spanning tree exists. We define $G$ to be the free group generated by edges $\left(v, v^{\prime}\right) \in \Delta$ modulo the relations

[R1]. $\left(v, v^{\prime}\right)=1$ if $\left(v, v^{\prime}\right) \in T$, and

[R2]. $\left(v, v^{\prime}\right)\left(v^{\prime}, v^{\prime \prime}\right)=\left(v, v^{\prime \prime}\right)$ if $\left\{v, v^{\prime}, v^{\prime \prime}\right\} \in \Delta$.

The following theorem will be crucial in our study of the fundamental group.

Theorem 3.2 ([10] III.7.3) With the above notation,

$$
E\left(\Delta, v_{0}\right) \approx G .
$$

We note for later use that this isomorphism is given by the map

$$
\Phi: E\left(\Delta, v_{0}\right) \rightarrow G
$$

that sends $\left[\left(v_{0}, v_{1}\right)\left(v_{1}, v_{2}\right) \cdots\left(v_{r-1}, v_{r}\right)\right]_{E} \mapsto\left[\left(v_{0}, v_{1}\right)\left(v_{1}, v_{2}\right) \cdots\left(v_{r-1}, v_{r}\right)\right]_{G}$. Here, $[-]_{E}$ and $[-]_{G}$ denote the equivalence classes of an edge path in $E\left(\Delta, v_{0}\right)$ and $G$, respectively. The inverse to this map is defined on the generators of $G$ as follows. For $\left(v, v^{\prime}\right) \in \Delta$, there is an edge path $\gamma$ from $v_{0}$ to $v$ along $T$ and an edge path $\gamma^{\prime}$ from $v^{\prime}$ to $v_{0}$ along $T$. Using these paths, we map $\Phi^{-1}\left[\left(v, v^{\prime}\right)\right]_{G}=\left[\gamma\left(v, v^{\prime}\right) \gamma^{\prime}\right]_{E}$. 


\section{The Fundamental Group and $h$-numbers}

Our goal now is to use Theorem 3.2 to bound the minimal number of generators of $\pi_{1}(\Delta, *)$. For ease of notation, let $m(\Delta, *)$ denote the minimal number of generators of $\pi_{1}(\Delta, *)$. When the basepoint is understood or irrelevant (e.g. when $\Delta$ is connected) we will write $m(\Delta)$ in place of $m(\Delta, *)$. For the remainder of this section, we will be concerned with simplicial complexes $\Delta$ of dimension $(d-1)$ with the following properties:

(I). $\Delta$ is pure,

(II). $\Delta$ is balanced,

(III). $\mathrm{lk}_{\Delta} F$ is connected for all faces $F \in \Delta$ with $0 \leq|F|<d-1$.

In particular, property (III) implies that $\Delta$ is connected by taking $F$ to be the empty face.

Since results on balanced simplicial complexes are well-suited to proofs by induction, we begin with the following observation.

Proposition 4.1 Let $\Delta$ be a simplicial complex with $d \geq 2$ that satisfies properties (I)(III). If $F \in \Delta$ is a face with $|F|<d-1$, then $\mathrm{lk}_{\Delta} F$ satisfies properties (I)-(III) as well.

Proof: When $d=2$, the result holds trivially since the only such face $F$ is the empty face. When $d>3$ and $F$ is nonempty, it is sufficient to show that the result holds for a single vertex $v \in F$. Indeed, if we set $G=F \backslash\{v\}$, then $\mathrm{lk}_{\Delta} F=\mathrm{lk}_{\mathrm{lk}_{\Delta} v} G$ at which point we may appeal to induction on $|F|$.

We immediately see that $\mathrm{lk}_{\Delta} v$ inherits properties (I) and (II) from $\Delta$. Finally, if $\sigma \in \mathrm{lk}_{\Delta} v$ is a face with $|\sigma|<d-2$, then $\operatorname{lk}_{\mathrm{lk}_{\Delta} v} \sigma=\mathrm{lk}_{\Delta}(\sigma \cup v)$ is connected by property (III).

Lemma 4.2 Let $\Delta$ be a $(d-1)$-dimensional simplicial complex with $d \geq 2$ that satisfies properties (I) and (III). If $F$ and $F^{\prime}$ are facets in $\Delta$, then there is a chain of facets

$$
F=F_{0}, F_{1}, \ldots, F_{m}=F^{\prime}
$$

such that $\left|F_{i} \cap F_{i+1}\right|=d-1$ for all $i$.

Remark 4.3 We say that a pure simplicial complex satisfying property (*) is strongly connected.

Proof: $\quad$ We proceed by induction on $d$. When $d=2, \Delta$ is a connected graph, and such a chain of facets is a path from some vertex $v \in F$ to a vertex $v^{\prime} \in F^{\prime}$. We now assume that $d \geq 3$.

First, we note that the closed star of each face in $\Delta$ is strongly connected. Indeed, by induction the link (and hence the closed $\operatorname{star} \overline{\mathrm{st}}_{\Delta} \sigma$ ) of each face $\sigma \in \Delta$ with $|\sigma|<d-1$ 
is strongly connected. On the other hand, if $\sigma \in \Delta$ is a face with $|\sigma|=d-1$, then every facet in $\overline{\mathrm{st}}_{\Delta} \sigma$ contains $\sigma$ and so $\overline{\mathrm{st}}_{\Delta} \sigma$ is strongly connected as well. Finally, if $\sigma$ is a facet, then $\overline{\mathrm{st}}_{\Delta} \sigma$ is strongly connected as it only contains a single facet.

It is also clear that if $\Delta^{\prime}$ and $\Delta^{\prime \prime}$ are strongly connected subcomplexes of $\Delta$ such that $\Delta^{\prime} \cap \Delta^{\prime \prime}$ contains a facet, then $\Delta^{\prime} \cup \Delta^{\prime \prime}$ is strongly connected as well. Finally, suppose $\Delta_{0} \subseteq \Delta$ is a maximal strongly connected subcomplex of $\Delta$. If $F \in \Delta_{0}$ is any face, then $\overline{\mathrm{st}}_{\Delta} F$ intersects $\Delta_{0}$ in a facet. Since $\overline{\mathrm{st}}_{\Delta} F \cup \Delta_{0}$ is strongly connected and $\Delta_{0}$ is maximal, we must have $\overline{\operatorname{st}}_{\Delta} F \subseteq \Delta_{0}$. Thus $\Delta_{0}$ is a connected component of $\Delta$. Since $\Delta$ is connected, $\Delta=\Delta_{0}$.

Lemma 4.4 Let $\Delta$ be a $(d-1)$-dimensional simplicial complex with $d \geq 2$ that satisfies properties (I)-(III). For any $S \subseteq[d]$ with $|S|=2$, the rank selected subcomplex $\Delta_{S}$ is connected.

Proof: $\quad$ Say $S=\left\{c_{1}, c_{2}\right\}$. Pick vertices $v, v^{\prime} \in \Delta_{S}$ and facets $F \ni v, F^{\prime} \ni v^{\prime}$. By Lemma 4.2 , there is a chain of facets $F=F_{1}, \ldots, F_{m}=F^{\prime}$ for which $F_{i}$ intersects $F_{i+1}$ in a codimension 1 face. We claim that a path from $v$ to $v^{\prime}$ in $\Delta_{S}$ can be found in $\cup_{i=1}^{m} F_{i}$.

When $m=1,\left\{v, v^{\prime}\right\}$ is an edge in $F=F_{1}=F^{\prime}$. For $m>1$, we examine the facet $F_{1}$. Without loss of generality, say $\kappa(v)=c_{1}$, and let $w \in F_{1}$ be the vertex with $\kappa(w)=c_{2}$. If $\{v, w\} \in F_{1} \cap F_{2}$, then the facet $F_{1}$ in our chain is extraneous, and we could have taken $F=F_{2}$ instead. Inductively, we can find a path from $v$ to $v^{\prime}$ in $\Delta_{S}$ that is contained in $\cup_{i=2}^{m} F_{i}$. On the other hand, if $v \notin F_{2}$, then we can find a path from $w$ to $v^{\prime}$ in $\Delta_{S}$ that is contained in $\cup_{i=2}^{m} F_{i}$ by induction. Since $(v, w) \in \Delta_{S}$, this path extends to a path from $v$ to $v^{\prime}$ in $\Delta_{S}$.

Theorem 4.5 Let $\Delta$ be a $(d-1)$-dimensional simplicial complex with $d \geq 2$ that satisfies properties (I)-(III), and $S \subseteq[d]$ with $|S|=2$. If $v$, $v^{\prime}$ are vertices in $\Delta_{S}$, then any edge path $\gamma$ from $v$ to $v^{\prime}$ in $\Delta$ is equivalent to an edge path from $v$ to $v^{\prime}$ in $\Delta_{S}$.

Proof: $\quad$ When $d=2, \Delta_{S}=\Delta$, and the result holds trivially, so we can assume $d \geq 3$.

We may write our edge path $\gamma$ as a sequence

$$
\gamma=\left(v_{0}, v_{1}\right)\left(v_{1}, v_{2}\right) \cdots\left(v_{r-1}, v_{r}\right)
$$

where $v_{0}=v, v_{r}=v^{\prime}$, and $\left\{v_{i}, v_{i+1}\right\} \in \Delta$ for all $i$. We establish the claim by induction on $r$. When $r=1$, the edge $\left(v, v^{\prime}\right)$ is already an edge in $\Delta_{S}$. Now we assume $r>1$. If $v_{1} \in \Delta_{S}$, the sequence $\left(v_{1}, v_{2}\right) \cdots\left(v_{r-1}, v_{r}\right)$ is equivalent to an edge path $\widetilde{\gamma}$ from $v_{1}$ to $v^{\prime}$ in $\Delta_{S}$ by our induction hypothesis on $r$. Hence $\gamma$ is equivalent to $\left(v_{0}, v_{1}\right) \widetilde{\gamma}$.

On the other hand, suppose that $v_{1} \notin \Delta_{S}$. Since $\kappa\left(v_{1}\right) \notin S$ and $\Delta$ is pure and balanced, there is a vertex $\widetilde{v} \in \Delta_{S}$ such that $\left\{v_{1}, v_{2}, \widetilde{v}\right\} \in \Delta$. By Proposition $4.1, \mathrm{lk}_{\Delta} v_{1}$ is a simplicial complex of dimension at least 1 satisfying properties (I)-(III). Thus by Lemma 4.4 , there is an edge path $\gamma^{\prime}=\left(u_{0}, u_{1}\right) \cdots\left(u_{k-1}, u_{k}\right)$ such that $u_{0}=v_{0}, u_{k}=\widetilde{v}$, and each edge $\left\{u_{i}, u_{i+1}\right\} \in\left(\mathrm{lk}_{\Delta} v_{1}\right)_{S}$. Since each edge $\left\{u_{i}, u_{i+1}\right\} \in \mathrm{lk}_{\Delta} v_{1}$, it follows that $\left\{u_{i}, u_{i+1}, v_{1}\right\} \in \Delta$ for all $i$. 
We now use the fact that $\left(u, u^{\prime}\right)\left(u^{\prime}, u^{\prime \prime}\right) \sim\left(u, u^{\prime \prime}\right)$ for all $\left\{u, u^{\prime}, u^{\prime \prime}\right\} \in \Delta$ to see the following simple equivalences of edge paths.

$$
\begin{aligned}
\left(v_{0}, v_{1}\right)\left(v_{1}, \widetilde{v}\right)= & \left(u_{0}, v_{1}\right)\left(v_{1}, \widetilde{v}\right) \\
\sim & \left(u_{0}, u_{1}\right)\left(u_{1}, v_{1}\right)\left(v_{1}, \widetilde{v}\right) \\
\sim & \left(u_{0}, u_{1}\right)\left(u_{1}, u_{2}\right)\left(u_{2}, v_{1}\right)\left(v_{1}, \widetilde{v}\right) \\
& \cdots \\
\sim & \left(u_{0}, u_{1}\right)\left(u_{1}, u_{2}\right) \cdots\left(u_{k-2}, u_{k-1}\right)\left(u_{k-1}, v_{1}\right)\left(v_{1}, \widetilde{v}\right) \\
\sim & \left(u_{0}, u_{1}\right)\left(u_{1}, u_{2}\right) \cdots\left(u_{k-2}, u_{k-1}\right)\left(u_{k-1}, \widetilde{v}\right) .
\end{aligned}
$$

For convenience, we write $\gamma_{1}=\left(u_{0}, u_{1}\right)\left(u_{1}, u_{2}\right) \cdots\left(u_{k-2}, u_{k-1}\right)\left(u_{k-1}, \widetilde{v}\right)$. Now we observe that $\left(v_{0}, v_{1}\right)\left(v_{1}, v_{2}\right) \sim\left(v_{0}, v_{1}\right)\left(v_{1}, \widetilde{v}\right)\left(\widetilde{v}, v_{2}\right)$ so that

$$
\begin{aligned}
\gamma & =\left(v_{0}, v_{1}\right)\left(v_{1}, v_{2}\right)\left(v_{2}, v_{3}\right) \cdots\left(v_{r-1}, v_{r}\right) \\
& \sim\left(v_{0}, v_{1}\right)\left(v_{1}, \widetilde{v}\right)\left(\widetilde{v}, v_{2}\right)\left(v_{2}, v_{3}\right) \cdots\left(v_{r-1}, v_{r}\right) \\
& \sim \gamma_{1}\left(\widetilde{v}, v_{2}\right)\left(v_{2}, v_{3}\right) \cdots\left(v_{r-1}, v_{r}\right) .
\end{aligned}
$$

By induction on $r$, there is an edge path $\gamma_{2}$ in $\Delta_{S}$ from $\widetilde{v}$ to $v_{r}$ that is equivalent to $\left(\widetilde{v}, v_{2}\right)\left(v_{2}, v_{3}\right) \cdots\left(v_{r-1}, v_{r}\right)$ so that $\gamma \sim \gamma_{1} \gamma_{2}$. Thus, indeed, $\gamma$ is equivalent to an edge path in $\Delta_{S}$.

Setting $v=v^{\prime}=v_{0}$, we have the following corollary.

Corollary 4.6 If $v_{0} \in \Delta_{S}$, every class in $E\left(\Delta, v_{0}\right)$ can be represented by a closed edge path in $\Delta_{S}$.

Now we have an explicit description of a smaller generating set of $\pi_{1}\left(\Delta, v_{0}\right)$.

Lemma 4.7 Let $\Delta$ be a $(d-1)$-dimensional simplicial complex with $d \geq 2$ that satisfies properties (I)-(III). For a fixed $S \subseteq[d]$ with $|S|=2$, the group $G$ of Theorem 3.2 is generated by the edges $\left(v, v^{\prime}\right)$ with $\left\{v, v^{\prime}\right\} \in \Delta_{S}$.

Proof: In order to use Theorem 3.2, we must choose some spanning tree $T$ in the 1skeleton of $\Delta$. We will do this in a specific way. Since $\Delta_{S}$ is a connected graph, we can find a spanning tree $\widetilde{T}$ in $\Delta_{S}$. Since $\Delta$ is connected, we can extend $\widetilde{T}$ to a spanning tree $T$ in $\Delta$ so that $\widetilde{T} \subseteq T$.

By Corollary 4.6 , each class in $E\left(\Delta, v_{0}\right)$ is represented by a closed edge path in $\Delta_{S}$, and hence the isomorphism $\Phi$ of Theorem 3.2 maps $E\left(\Delta, v_{0}\right)$ into the subgroup of $H \subseteq G$ generated by edges $\left(v, v^{\prime}\right) \in \Delta_{S}$. Since $\Phi$ is surjective, we must have $H=G$.

Corollary 4.8 With $\Delta$ and $S$ as in Lemma 4.7, we have

$$
m(\Delta) \leq h_{2}\left(\Delta_{S}\right)
$$


Proof: $\quad$ Lemma 4.7 tells us that the $f_{1}\left(\Delta_{S}\right)$ edges in $\Delta_{S}$ generate the group $G$. Since our spanning tree $T$ contains a spanning tree in $\Delta_{S}, f_{0}\left(\Delta_{S}\right)-1$ of these generators will be identified with the identity. Thus

$$
m(\Delta) \leq f_{1}\left(\Delta_{S}\right)-f_{0}\left(\Delta_{S}\right)+1=h_{2}\left(\Delta_{S}\right)
$$

While the proof of the above corollary requires specific information about the set $S$ and a specific spanning tree $T \subset \Delta$, its result is purely combinatorial. Since $\Delta$ is connected, $\pi_{1}(\Delta, *)$ is independent of the basepoint, and so we can sum over all such sets $S \subset[d]$ with $|S|=2$ to get

$$
\begin{aligned}
\left(\begin{array}{l}
d \\
2
\end{array}\right) m(\Delta) & \leq \sum_{|S|=2} h_{2}\left(\Delta_{S}\right) \\
& =h_{2}(\Delta) \quad \text { by Equation }(1) .
\end{aligned}
$$

This gives the following theorem.

Theorem 4.9 Let $\Delta$ be a pure, balanced simplicial complex of dimension $(d-1)$ with the property that $\operatorname{lk}_{\Delta} F$ is connected for all faces $F \in \Delta$ with $|F|<d-1$. Then

$$
\left(\begin{array}{l}
d \\
2
\end{array}\right) m(\Delta) \leq h_{2}(\Delta)
$$

\section{$5 \quad$ Extensions and Further Questions}

\subsection{Simplicial Posets}

We now generalize the results in Section 4 to the class of simplicial posets. A simplicial poset is a poset $P$ with a least element $\hat{0}$ such that for any $x \in P \backslash\{\hat{0}\}$, the interval $[\hat{0}, x]$ is a Boolean algebra (see Björner [1] or Stanley [12]). That is to say that the interval $[\hat{0}, x]$ is isomorphic to the face poset of a simplex. Thus $P$ is graded by $\operatorname{rk}(\sigma)=k+1$ if $[\hat{0}, \sigma]$ is isomorphic to the face poset of a $k$-simplex. The face poset of a simplicial complex is a simplicial poset. Following [1], we see that every simplicial poset $P$ has a geometric interpretation as the face poset of a regular CW-complex $|P|$ in which each cell is a simplex and each pair of simplices is joined along a possibly empty subcomplex of their boundaries. We call $|P|$ the realization of $P$. With this geometric picture in mind, we refer to elements of $P$ as faces and work interchangeably between $P$ and $|P|$. In particular, we refer to rank- 1 elements of $P$ as vertices and maximal rank elements of $P$ as facets. As in the case of simplicial complexes, we say that the dimension of a face $\sigma \in P$ is $\operatorname{rk}(\sigma)-1$, and the dimension of $P$ is $d-1$ where $d=\operatorname{rk}(P)=\max \{\operatorname{rk}(\sigma): \sigma \in P\}$. We say that $P$ is pure if each of its facets has the same rank. In addition, we can form the order complex $\Delta(\bar{P})$ of the poset $\bar{P}=P \backslash\{\hat{0}\}$, which gives a barycentric subdivision of $|P|$. 
As with simplicial complexes, we define the link of a face $\tau \in P$ as

$$
\operatorname{lk}_{P} \tau=\{\sigma \in P: \sigma \geq \tau\}
$$

It is worth noting that $l k_{P} \tau$ is a simplicial poset whose minimal element is $\tau$, but $\operatorname{lk}_{P} \tau$ is not necessarily a subcomplex of $|P|$. All hope is not lost, however, since for any saturated chain $F=\left\{\tau_{0}<\tau_{1}<\ldots<\tau_{r}=\tau\right\}$ in $(\hat{0}, \tau]$ we have $\operatorname{lk}_{\Delta(\bar{P})}(F) \cong \Delta\left(\overline{\operatorname{lk}_{P}(\tau)}\right)$. Here we say $F$ is saturated if each relation $\tau_{i}<\tau_{i+1}$ is a covering relation in $P$.

We are also concerned with balanced simplicial posets and strongly connected simplicial posets. Suppose $P$ is a pure simplicial poset of dimension $(d-1)$, and let $V$ denote the vertex set of $P$. We say that $P$ is balanced if there is a coloring $\kappa: V \rightarrow[d]$ such that for each facet $\sigma \in P$ and distinct vertices $v, w<\sigma$, we have $\kappa(v) \neq \kappa(w)$. If $S \subseteq[d]$, we can form the $S$-rank selected poset of $P$, defined as

$$
P_{S}=\{\sigma \in P: \kappa(\sigma) \subseteq S\} \text { where } \kappa(\sigma)=\{\kappa(v): v<\sigma, \operatorname{rk}(v)=1\} .
$$

We say that $P$ is strongly connected if for all facets $\sigma, \sigma^{\prime} \in P$ there is a chain of facets

$$
\sigma=\sigma_{0}, \sigma_{1}, \ldots, \sigma_{m}=\sigma^{\prime}
$$

and faces $\tau_{i}$ of rank $d-1$ such that $\tau_{i}$ is covered by $\sigma_{i}$ and $\sigma_{i+1}$ for all $0 \leq i \leq m-1$. For simplicial complexes, the face $\tau_{i}$ is naturally $\sigma_{i} \cap \sigma_{i+1}$; however, for simplicial posets, the face $\tau_{i}$ is not necessarily unique.

As in Section 4, we are concerned with simplicial posets $P$ of rank $d$ satisfying the following three properties:

(i). $P$ is pure,

(ii). $P$ is balanced,

(iii). $\operatorname{lk}_{P} \sigma$ is connected for all faces $\sigma \in P$ with $0 \leq \operatorname{rk}(\sigma)<d-1$.

Our first task is to understand the fundamental group of a simplicial poset by constructing an analogue of the edge-path group of a simplicial complex. We have to be careful because there can be several edges connecting a given pair of vertices. An edge in $P$ is an oriented rank-2 element $e \in P$ with an initial vertex, denoted init $(e)$, and a terminal vertex, denoted term $(e)$. If $e$ is an edge, we let $e^{-1}$ denote its inverse edge, that is, we interchange the initial and terminal vertices of $e$, reversing the orientation of $e$. We note that the initial and terminal vertices of $e$ are distinct since $[\hat{0}, e]$ is a Boolean algebra. We also allow for the degenerate edge $e=(v, v)$ for any vertex $v \in P$. An edge path $\gamma$ in $P$ is a finite nonempty sequence $e_{0} e_{1} \cdots e_{r}$ of edges in $P \operatorname{such}$ that $\operatorname{term}\left(e_{i}\right)=\operatorname{init}\left(e_{i+1}\right)$ for all $0 \leq i \leq r-1$. A closed edge path at $v$ is an edge path $\gamma$ such that $\operatorname{init}\left(e_{0}\right)=v=\operatorname{term}\left(e_{r}\right)$. Given edge paths $\gamma$ from $v$ to $v^{\prime}$ and $\gamma^{\prime}$ from $v^{\prime}$ to $v^{\prime \prime}$, we can form their product edge path $\gamma \gamma^{\prime}$ from $v$ to $v^{\prime \prime}$ by concatenation.

Suppose $\sigma \in P$ is a rank-3 face with (distinct) vertices $v, v^{\prime}$ and $v^{\prime \prime}$ and edges $e, e^{\prime}$ and $e^{\prime \prime}$ with $\operatorname{init}(e)=v=\operatorname{init}\left(e^{\prime \prime}\right), \operatorname{init}\left(e^{\prime}\right)=v^{\prime}=\operatorname{term}(v)$ and $\operatorname{term}\left(e^{\prime \prime}\right)=v^{\prime \prime}=\operatorname{term}\left(e^{\prime}\right)$. Analogously to Section 3, we say that two edge paths $\gamma$ and $\gamma^{\prime}$ are simply equivalent if the unordered pair $\left\{\gamma, \gamma^{\prime}\right\}$ is equal to one of the following unordered pairs: 
- $\left\{e^{\prime \prime}, e e^{\prime}\right\}$ or $\left\{(v, v), e e^{-1}\right\}$;

- $\left\{\gamma_{1} e^{\prime \prime}, \gamma_{1} e e^{\prime}\right\}$ or $\left\{\gamma_{1}, \gamma_{1} e e^{-1}\right\}$ for some edge path $\gamma_{1}$ ending at $v$;

- $\left\{e^{\prime \prime} \gamma_{2}, e e^{\prime} \gamma_{2}\right\}$ or $\left\{\gamma_{2},\left(e^{\prime}\right)^{-1} e^{\prime} \gamma_{2}\right\}$ for some edge path $\gamma_{2}$ starting at $v^{\prime \prime}$;

- $\left\{\gamma_{1} e^{\prime \prime} \gamma_{2}, \gamma_{1} e e^{\prime} \gamma_{2}\right\}$ for edge paths $\gamma_{1}, \gamma_{2}$ as above.

We say that two edge paths $\gamma$ and $\gamma^{\prime}$ are equivalent and write $\gamma \sim \gamma^{\prime}$ if there is a finite sequence of edge paths $\gamma=\gamma_{0}, \ldots, \gamma_{s}=\gamma^{\prime}$ such that $\gamma_{i}$ is simply equivalent to $\gamma_{i+1}$ for all $i$. As in the case of simplicial complexes, this forms an equivalence relation on the collection of edge paths in $P$ with initial vertex $v$ and terminal vertex $v^{\prime}$. We pick a base vertex $v_{0}$ and let $\widetilde{E}\left(P, v_{0}\right)$ denote the collection of equivalence classes of closed edge paths in $P$ at $v_{0}$. We give $\widetilde{E}\left(P, v_{0}\right)$ a group structure by loop multiplication, and the resulting group is called the edge path group of $P$ based at $v_{0}$.

Now we ask if the groups $\pi_{1}\left(P, v_{0}\right)$ and $\widetilde{E}\left(P, v_{0}\right)$ are isomorphic. As topological spaces, $|P|$ and $\Delta(\bar{P})$ are homeomorphic and so their fundamental groups are isomorphic. The latter space is a simplicial complex, and so we know that $E\left(\Delta(\bar{P}), v_{0}\right) \approx \pi_{1}\left(P, v_{0}\right)$. The following theorem will show that indeed $\pi_{1}\left(P, v_{0}\right) \approx \widetilde{E}\left(P, v_{0}\right)$.

Theorem 5.1 Let $P$ be a simplicial poset of rank d satisfying properties $(i)$ and (iii). If $v_{0}$ is a vertex in $P$, then

$$
\widetilde{E}\left(P, v_{0}\right) \approx E\left(\Delta(\bar{P}), v_{0}\right) .
$$

Proof: Given an edge $e \in P$ with initial vertex $v$ and terminal vertex $v^{\prime}$, we define an edge path in $\Delta(\bar{P})$ from $v$ to $v^{\prime}$ by barycentric subdivision as $\operatorname{Sd}(e)=(v, e)\left(e, v^{\prime}\right)$. We define $\Phi: \widetilde{E}\left(P, v_{0}\right) \rightarrow E\left(\Delta(\bar{P}), v_{0}\right)$ by

$$
\Phi\left(\left[e_{0} e_{1} \cdots e_{r}\right]_{\widetilde{E}}\right)=\left[\operatorname{Sd}\left(e_{0}\right) \operatorname{Sd}\left(e_{1}\right) \cdots \operatorname{Sd}\left(e_{r}\right)\right]_{E} .
$$

It is easy to check that $\Phi$ is well-defined, as it respects simple equivalences.

We now claim that $\Delta(\bar{P})$ in fact satisfies properties (I)-(III) of Section 4 . Since $\Delta(\bar{P})$ is the order complex of a pure poset, it is pure and balanced. Indeed, the vertices in $\Delta(\bar{P})$ are elements $\sigma \in \bar{P}$, colored by their rank in $P$. Finally, for a saturated chain $F=\left\{\tau_{1}<\tau_{2}<\ldots<\tau_{r}=\tau\right\}$ in $\bar{P}$ for which $r<d-1$, we see that $\operatorname{lk}_{\Delta(\bar{P})} F \cong \Delta\left(\overline{\operatorname{lk}_{P}(\tau)}\right)$ is connected since $\operatorname{lk}_{P} \tau$ is connected. By Proposition 3.3 in [4], we need only consider saturated chains here. By Theorem 4.7, it follows that any class in $E\left(\Delta(\bar{P}), v_{0}\right)$ can be represented by a closed edge path in $(\Delta(\bar{P}))_{\{1,2\}}$. In particular, we can represent any class in $E\left(\Delta(\bar{P}), v_{0}\right)$ by an edge path $\gamma=\operatorname{Sd}\left(e_{0}\right) \operatorname{Sd}\left(e_{1}\right) \cdots \operatorname{Sd}\left(e_{r}\right)$ for some edge path $e_{0} e_{1} \cdots e_{r}$ in $P$. This gives a well-defined inverse to $\Phi$.

With Theorem 5.1 and the above definitions, the proofs of Proposition 4.1, Lemmas 4.2 and 4.4 , and Theorem 4.5 carry over almost verbatim to the context of simplicial posets and can be used to prove the following Lemma.

Lemma 5.2 Let $P$ be a simplicial poset of rank $d \geq 2$ that satisfies properties (i)-(iii). 
a. If $\sigma \in P$ is a face and $\operatorname{rk}(\sigma)<d-1$, then $\operatorname{lk}_{P} \sigma$ satisfies properties (i)-(iii) as well.

b. $P$ is strongly connected.

c. For any $S \subseteq[d]$ with $|S|=2$, the rank selected subcomplex $P_{S}$ is connected.

$d$. If $v$ and $v^{\prime}$ are vertices in $P_{S}$, then any edge path $\gamma$ from $v$ to $v^{\prime}$ in $P$ is equivalent to an edge path from $v$ to $v^{\prime}$ in $P_{S}$.

As in Section 4, part (d) of this Lemma implies the following generalization of Theorem 4.9 .

Theorem 5.3 Let $P$ be a pure, balanced simplicial poset of rank $d$ with the property that $\mathrm{lk}_{P} \sigma$ is connected for each face $\sigma \in P$ with $\operatorname{rk}(\sigma)<d-1$. Then

$$
\left(\begin{array}{l}
d \\
2
\end{array}\right) m(P) \leq h_{2}(P) \text {. }
$$

\subsection{How Tight are the Bounds?}

We now turn our attention to a number of examples to determine if the bounds given by Theorems 4.9 and 5.3 are tight. We begin by studying a family of simplicial posets constructed by Novik and Swartz in [8]. Lemma 7.6 in [8] constructs a simplicial poset $X(1, d)$ of dimension $(d-1)$ satisfying properties (i)-(iii) whose geometric realization is a $(d-2)$-disk bundle over $\mathbb{S}^{1}$ and $h_{2}(X(1, d))=\left(\begin{array}{l}d \\ 2\end{array}\right)$. As $X$ is a bundle over $\mathbb{S}^{1}$ with contractible fiber, we have $\pi_{1}(X(1, d), *) \approx \mathbb{Z}$ so that $m(X(1, d))=1$. This construction shows that the bound in Theorem 5.3 is tight. Moreover, taking connected sums of $r$ copies of $X(1, d)$ (when $d \geq 4$ ) gives a simplicial poset $P$ whose fundamental group is isomorphic to $\mathbb{Z}^{r}$ and $h_{2}(P)=r\left(\begin{array}{l}d \\ 2\end{array}\right)$. We do not know, however, if the bound in Theorem 5.3 is tight when $\pi_{1}(P, *)$ is either non-free or non-Abelian. We would also like to know if Theorem 5.3 holds if we drop the condition that $P$ is balanced.

\section{Acknowledgements}

I am grateful to my advisor, Isabella Novik, for her guidance, and for carefully editing many preliminary drafts of this paper. I am also grateful to the anonymous referees who provided many helpful suggestions.

\section{References}

[1] A. Björner, Posets, regular CW complexes and Bruhat order, European J. Combin. 5 (1984), 7-16.

[2] A. Björner and G. Kalai, An extended Euler-Poincaré theorem, Acta Math. 161 (1988), 279-303. 
[3] A. Björner and G. Kalai, On $f$-vectors and homology, Combinatorial Mathematics, Proc. NY Academy of Science 555 (1989), 63-80.

[4] A. Duval, Free resolutions of simplicial posets, J. Algebra 188 (1997), 363-399.

[5] M. Goresky and R. MacPherson, Intersection homology theory. Topology 19 (1980), no. $2,135-162$.

[6] M. Jungerman and G. Ringel, Minimal triangulations on orientable surfaces. Acta Math. 145 (1980), 121-154.

[7] G. Kalai, Rigidity and the lower bound Theorem I, Invent. Math. 88 (1987), 125-151.

[8] I. Novik and E. Swartz, Socles of Buchsbaum modules, complexes and posets, arXiv:0711.0783.

[9] H. Seifert and W. Threlfall, A Textbook of Topology. Academic Press 1980, reprint of the German edition Lehrbuch der Topologie, 1934, Teubner.

[10] E. Spanier, Algebraic Topology. Corrected reprint, Springer-Verlag, New York-Berlin, 1981.

[11] R. Stanley, Balanced Cohen-Macaulay complexes, Trans. Amer. Math. Soc., Vol. 249, No. 1, (1979), pp. 139-157.

[12] R. Stanley, $f$-vectors and $h$-vectors of simplicial posets, J. Pure Applied Algebra 71 (1991), 319-331.

[13] R. Stanley, Combinatorics and Commutative Algebra, Boston Basel Berlin: Birkhäuser, 1996. 